time vs placebo from Week 4. Time to CRR was shorter in the $180 \mathrm{mg}$ group (17.3 weeks) vs placebo (20.4 weeks). The $180 \mathrm{mg}$ group also showed improvement vs placebo in total SLEDAI (SELENA) and its subscores.

The unexpected high placebo response prompted a post hoc analysis evaluating confirmed CRR (CCRR), whereby confirmation of the endpoint was required at both Weeks 46 (penultimate visit on treatment) and 52. A 15.2\% higher cCRR in the $180 \mathrm{mg}$ group $(44.3 \%)$ vs placebo $(29.1 \%)$ was observed $(p=0.26)$.

While based on a small sample size, there were more reports of infection-related severe and serious adverse events and neutropenia in the $240 \mathrm{mg}$ group compared with placebo. Of note, in those who experienced neutropenia, a clinical impact (e.g. increase in infections) was not established. Aside from these observations, safety data were comparable across treatment groups.

Larger decreases from baseline were observed in the percentage of CD27-lgD-CD95+, CD27-lgD+CD95+, CD27+lgD+CD95+ and CD27+lgD-CD95+ B-cell subsets in the 180 and $240 \mathrm{mg}$ groups compared with placebo.

Treatment-emergent anti-drug antibodies (ADAs) were detected in five patients treated with $\mathrm{BI} 655054$, all at low titre, and in one who received placebo; ADAs had no impact on pharmacokinetics or safety.

Conclusion: The trial did not meet its primary CRR endpoint. However, when confirmation of CRR was required at both Weeks 46 and 52, the resultant decrease in the placebo response generated an effect size of $15.2 \%$ and $9.1 \%$ in favour of $180 \mathrm{mg}$ and $240 \mathrm{mg} \mathrm{BI} 655064$, respectively.

Table 1. Efficacy endpoints at Week 52

\begin{tabular}{|c|c|c|c|c|}
\hline & \multirow[t]{2}{*}{ Placebo $(n=40)$} & \multicolumn{3}{|l|}{ BI 655064} \\
\hline & & $120 \mathrm{mg}(\mathrm{n}=21)$ & $180 \mathrm{mg}(\mathrm{n}=20)$ & $240 \mathrm{mg}(\mathrm{n}=40)$ \\
\hline Observed CRR, $\mathrm{n}$ & 20 & 8 & 9 & 18 \\
\hline Adjusted* CRR, \% & 48.3 & 38.3 & 45 & 44.6 \\
\hline Observed cCRR, n & 13 & 5 & 9 & 16 \\
\hline Adjusted $^{*}$ cCRR, \% & 29.1 & 22.5 & 44.3 & 38.2 \\
\hline \multicolumn{5}{|c|}{ Mean change from baseline in SLEDAI } \\
\hline Total score & -6.5 & -6.1 & -9.7 & -8.2 \\
\hline Non-renal score & -1.4 & -3.0 & -2.8 & -3.1 \\
\hline Renal score & -5.1 & -3.7 & -6.8 & -5.0 \\
\hline Clinical score & -5.7 & -3.9 & -7.9 & -6.5 \\
\hline
\end{tabular}

CRR based on 24h proteinuria; cCRR based on UP/UC (spot urine) at Weeks 46 and 52 . *Logistic regression model including treatment and the covariates race and proteinuria at screening.

Disclosure of Interests: David Jayne Consultant of: DRJ has received consulting fees from AstraZeneca, Boehringer Ingelheim, GlaxoSmithKline Research \& Development Ltd, Novartis, and Roche, Juergen Steffgen Employee of: JS is employed by Boehringer Ingelheim., Juanita Romero-Diaz Consultant of: JRD has received research consulting fees from Boehringer Ingelheim, Hirofumi Amano Grant/research support from: HA has received research grants from Boehringer Ingelheim, Kajohnsak Noppakun Consultant of: KN has received honoraria from Boehringer Ingelheim, Novartis, Roche, Jansen, AstraZeneca, Otsuka Pharmaceuticals, Astellas Pharma, Abbott, Sanofi and Novo Nordisk, Grant/research support from: KN has received research grants from Boehringer Ingelheim, GlaxoSmithKline Research \& Development Ltd, Visterra Inc., Kalbe Genexine Biologics, Aurinia Pharmaceuticals Inc., and Omeros Corporation., Harold Michael Gomez Speakers bureau: HMG has received speaker's fees for Pfizer, MSD, Unilab, Astellas Pharma, AstraZeneca, GlaxoSmithKline Research \& Development Ltd, and Aurinia Pharmaceuticals Inc., Rhona Recto: None declared, Valérie Belsack Employee of: Boehringer Ingelheim, Nora Fagan Employee of: Boehringer Ingelheim, Steven Padula Employee of: Boehringer Ingelheim, Ivette Revollo Employee of: Boehringer Ingelheim, Jing Wu Employee of: Boehringer Ingelheim, Sudha Visvanathan Employee of: Boehringer Ingelheim, Richard Furie Consultant of: RF has received research consulting fees, Grant/research support from: RF has received clinical trial support DOI: 10.1136/annrheumdis-2021-eular.1401

\section{POS0688 CHARACTERIZATION OF PK/PD OF ANIFROLUMAB IN PATIENTS WITH MODERATE TO SEVERE SLE}

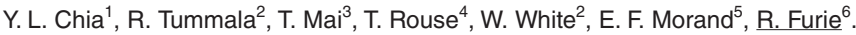
${ }^{1}$ AstraZeneca, BioPharmaceuticals R\&D, South San Francisco, United States of America; ${ }^{2}$ AstraZeneca, BioPharmaceuticals R\&D, Gaithersburg, United States of America; ${ }^{3}$ Genentech, Clinical Pharmacology and Modeling \& Simulation, South San Francisco, United States of America; ${ }^{4}$ AstraZeneca, BioPharmaceuticals R\&D, Gothenburg, Sweden; ${ }^{5}$ Monash University, Centre for Inflammatory Disease Monash Health, Melbourne, Australia; ${ }^{6}$ Zucker School of Medicine at Hofstra/Northwell, Division of Rheumatology, Great Neck, United States of America

Background: In the TULIP-1 and TULIP-2 trials, anifrolumab, a type I interferon (IFN) receptor antibody, at a dosage of $300 \mathrm{mg}$ once every 4 weeks (Q4W), demonstrated consistent median pharmacokinetic (PK) concentrations ${ }^{1}$ and sustained neutralization of the pharmacodynamic (PD) 21-gene type I IFN gene signature (IFNGS) $)^{2-4}$ in patients with moderate to severe systemic lupus erythematosus (SLE) despite standard therapy.

Objectives: To characterize the PK/PD relationship of anifrolumab and to confirm anifrolumab $300 \mathrm{mg}$ provides adequate PD neutralization in IFNGS testhigh patients.

Methods: This study included IFNGS test-high patients from the phase 3 randomized, placebo-controlled, 52-week TULIP-1 ${ }^{2}$ (NCT02446912) and TULIP-2 ${ }^{3}$ (NCT02446899) trials of intravenous anifrolumab $150 \mathrm{mg}$ or $300 \mathrm{mg}$ Q4W plus standard therapy. IFNGS test status (high or low) at screening was classified with an analytically validated 4-gene GPCR based test on whole blood. ${ }^{2}$ PD neutralization was measured with 21-gene type I IFNGS and expressed as a percentage change from baseline. ${ }^{3}$ For the graphic PK/PD analysis, patients with $\geq 1$ quantifiable serum PK sample and $\geq 1$ PD measurement before discontinuation were categorized depending on $\mathrm{C}_{\text {ave }}$ (individual predicted average anifrolumab concentration over treatment duration) median and tertiles $(T)$ for anifrolumab $150 \mathrm{mg}$ and $300 \mathrm{mg}$, respectively. Median PD IFNGS neutralization and medium absolute deviations were compared across $\mathrm{C}_{\text {ave }}$ subgroups. PK/PD modeling was assessed in patients with $\geq 1$ quantifiable serum PK sample and a baseline and $\geq 1$ postbaseline PD measurement before discontinuation, using a nonlinear mixed-effects model (NONMEM; version 7.3; ICON) to estimate parameters and characterize the PK/PD data. The PD/efficacy analysis included patients with $\geq 1$ postbaseline PD measurement before discontinuation. BILAG-based Combined Lupus Assessment (BICLA) response rates at Week (W)52 were compared across median PD neutralization quartiles $(\mathrm{Q})$ for pooled anifrolumab $300 \mathrm{mg}$ and $150 \mathrm{mg}$ groups.

Results: The PK/PD graphic analysis included 654 IFNGS test-high patients (placebo [ $\mathrm{n}=293]$; anifrolumab $150 \mathrm{mg}[\mathrm{n}=72]$ or $300 \mathrm{mg}[\mathrm{n}=289]$ ). $\mathrm{C}_{\text {eve }}$ was generally higher with anifrolumab $300 \mathrm{mg}(\mu \mathrm{g} / \mathrm{mL}$, TULIP-1: T1 $<32$, T2 32-<44.3, T3 $\geq 44.3$; TULIP-2: T1 <32.4, T2 32.4-<47.9, T3 $\geq 47.9$ ) than with anifrolumab $150 \mathrm{mg}$ (median $11.5 \mu \mathrm{g} / \mathrm{mL}$ ); overlap between anifrolumab $300 \mathrm{mg}$ and $150 \mathrm{mg} \mathrm{C}$ ave subgroups was small owing to nonlinearity. Anifrolumab $300 \mathrm{mg}$ elicited rapid

Figure. Observed PD neutralization of the 21-gene type I IFNGS according to $C_{\text {amo }}$ subgroup over the 52-week treatment duration in A) TULIP-1 and B) TULIP-2
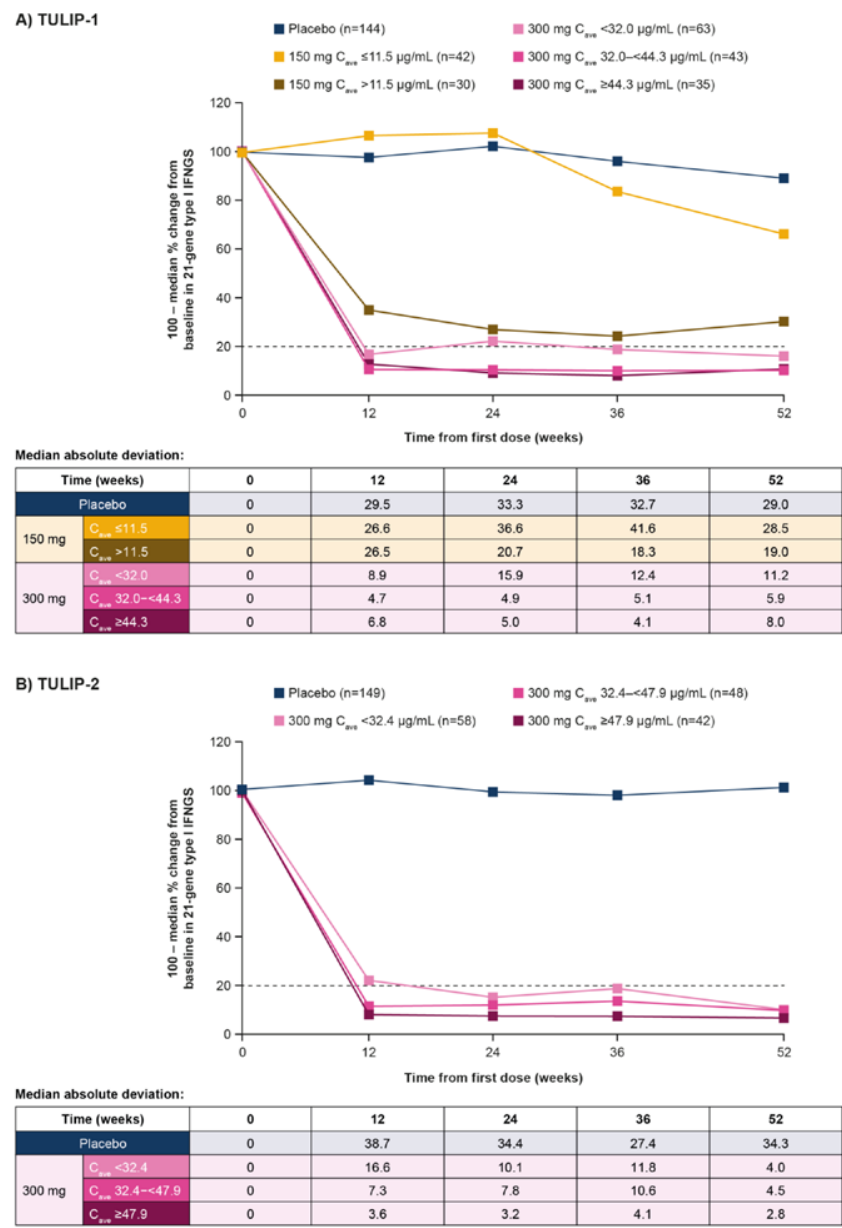

C..., average anifrolumab concentration over the treatment period; IFNGS, interferon gene signature; PD, pharmacodynam PK., pharmacokinetic. Figure includes patients with 21 quantifiable serum PK observation and 21 PD, measurement prior to discontinuation Po collected after discontinuations were not tincluded. 
(by $\mathrm{W}^{4}$ ) and sustained median PD neutralization $>80 \%$, vs a lower and delayed PD neutralization (median $>50 \%$ at W52) with anifrolumab $150 \mathrm{mg}$, and minimal PD neutralization with placebo. The median PD neutralization increased with higher $\mathrm{C}_{\text {vve }}$ subgroups, plateauing at $\sim 90 \%$ at W12-W52. All anifrolumab $300 \mathrm{mg}$ $\mathrm{C}_{\text {ave }}$ tertiles had a median PD neutralization $\sim 80 \%$; however, the variability was greater in the lowest $\mathrm{C}_{\text {ave }}$ tertiles vs higher $\mathrm{C}_{\text {ave }}$ tertiles across trials (Figure 1). The PK/PD modeling, which included 646 IFNGS test-high patients (placebo $[\mathrm{n}=289]$, anifrolumab $150 \mathrm{mg}[\mathrm{n}=70]$ or $300 \mathrm{mg}[\mathrm{n}=287])$, gave an IC $\mathrm{B}_{80}$ estimate of $3.88 \mu \mathrm{g} / \mathrm{mL}$. The median W24 (study midpoint) $\mathrm{C}_{\text {trough }}$ was higher with anifrolumab $300 \mathrm{mg}$ vs $150 \mathrm{mg}$ ( $15.6 \mathrm{vs} 0.2 \mu \mathrm{g} / \mathrm{mL}$ ); thus, the W24 $\mathrm{C}_{\text {trough }}$ exceeded the $I_{80}$ in a higher proportion of patients treated with anifrolumab $300 \mathrm{mg}$ vs $150 \mathrm{mg}$ ( $83 \%$ vs $\sim 27 \%)$. The PD/efficacy analysis included 341 patients who received anifrolumab. Higher median percentage PD neutralization quartiles (Q1 $<51.7 \%$, Q2 51.7\%-85.3\%, Q3 85.3\%-92.6\%, Q4 >92.6\%) were associated with higher W52 BICLA response rates (Q1 37.6\%, Q2 49.4\%, Q3 51.8\%, Q4 58.1\%).

Conclusion: In TULIP-1 and TULIP-2, anifrolumab $300 \mathrm{mg}$ yielded higher anifrolumab C vs $150 \mathrm{mg}$. High C was associated with rapid (W4 $\left.4^{4}-\mathrm{W} 12\right)$, substantial, and sustained PD neutralization of the 21-gene IFNGS in IFNGS test-high patients, which in turn was associated with higher efficacy.

REFERENCES:

[1] Kuruvilla D. Poster 360, AAPS 2020

[2] Furie RA. Lancet Rheumatol. 2019;1:e208-19.

[3] Morand EF. N Engl J Med. 2020;382:211-21.

[4] Furie R. Arthritis Rheumatol. 2017;69:379-86.

Acknowledgements: Writing assistance by Matilda Shackley, MPhil, of JK Associates Inc., part of Fishawack Health. This study was sponsored by AstraZeneca.

Disclosure of Interests: Yen Lin Chia Employee of: AstraZeneca, Raj Tummala Employee of: AstraZeneca, Tu Mai Employee of: Genentech, Tomas Rouse Employee of: AstraZeneca, Wendy White Employee of: AstraZeneca, Eric F. Morand Speakers bureau: AstraZeneca, Consultant of: AstraZeneca, Grant/ research support from: AstraZeneca, Richard Furie Consultant of: AstraZeneca, Grant/research support from: AstraZeneca

DOI: 10.1136/annrheumdis-2021-eular.1464

\begin{tabular}{|l|l|}
\hline POS0689 & A 6-MONTH OPEN-LABEL EXTENSION STUDY OF \\
THE SAFETY AND EFFICACY OF INTRAVENOUS \\
BELIMUMAB IN PATIENTS WITH LUPUS NEPHRITIS
\end{tabular}

R. Furie ${ }^{1}$, B. H. Rovin ${ }^{2}$, F. Houssiau ${ }^{3}$, G. Contreras ${ }^{4}$, P. Curtis ${ }^{5}$, A. Madan ${ }^{6}$, A. Jones-Leone ${ }^{7}$, M. Okily ${ }^{8}$, D. Roth ${ }^{9} .{ }^{1}$ Northwell Health, Division of Rheumatology, Great Neck, NY, United States of America; ${ }^{2}$ The Ohio State University, Division of Nephrology, Columbus, $\mathrm{OH}$, United States of America; ${ }^{3}$ Cliniques Universitaires Saint-Luc, Pôle de Pathologies, Brussels, Belgium; ${ }^{4}$ University of Miami Miller School of Medicine, Division of Nephrology, Division of Hypertension, Department of Medicine, Miami, FL, United States of America; ${ }^{5}$ GlaxoSmithKline, Department of Quantitative Sciences, Brentford, United Kingdom; ${ }^{6}$ GlaxoSmithKline, Clinical Sciences, Collegeville, PA, United States of America; ${ }^{7}$ GlaxoSmithKline, Medical Affairs, Collegeville, PA, United States of America; ${ }^{8}$ GlaxoSmithKline, $R \& D$ ImmunoInflammation, Collegeville, $P A$, United States of America; ${ }^{9}$ GlaxoSmithKline, Research and Development, Collegeville, PA, United States of America

Background: BLISS-LN (GSK Study BEL114054; NCT01639339), the largest lupus nephritis (LN) study to date, showed that intravenous (IV) belimumab (BEL) + standard therapy (ST) improved outcomes compared with ST alone in patients (pts) with active LN.

Objectives: To assess additional safety and efficacy data of BEL $+\mathrm{ST}$ in pts with $\mathrm{LN}$ in a 6-month open-label (OL) phase beyond 2 years of double-blind (DB) treatment in BLISS-LN.

Methods: In this OL phase, eligible completers of the DB phase received monthly BEL $10 \mathrm{mg} / \mathrm{kg}$ IV + ST for 6 months. Endpoints: safety; Primary Efficacy Renal Response (PERR; uPCR $\leq 0.7$; eGFR no worse than $20 \%$ below OL baseline eGFR or $\geq 60 \mathrm{ml} / \mathrm{min} / 1.73 \mathrm{~m}^{2}$; no prohibited medications) and Complete Renal Response (CRR; uPCR $<0.5$; eGFR no worse than $10 \%$ below OL baseline eGFR or $\geq 90 \mathrm{ml} / \mathrm{min} / 1.73 \mathrm{~m}^{2}$; no prohibited medications) at OL Week 28 proportion of pts with SLEDAI score $<4$; corticosteroid use; biomarkers. Analyses were based on observed data and summarised relative to the OL baseline (last value measured prior to the first dose of $\mathrm{OL}$ treatment).

Results: We enrolled 257 pts (57.4\% of pts in BEL114054) and treated 255 pts. All treated pts were included in the safety population (123 pts switched from placebo [PBO] to BEL; 132 pts remained on BEL). Efficacy was assessed in the safety population, excluding 1 pt due to non-compliance (mITT population; PBO to BEL: 122 pts; BEL to BEL: 132 pts). $96.5 \%$ of pts completed the OL phase; $3.5 \%$ withdrew, mainly due to adverse events (AE; $2.0 \%$ ).

Overall, 168/255 (65.9\%) pts had $\geq 1$ AE (76/123 [61.8\%] PBO to BEL pts; $92 / 132$ [69.7\%] BEL to BEL pts); $15 / 255$ (5.9\%) pts had $\geq 1$ serious AE (5/123 [4.1\%] PBO to BEL pts; $10 / 132$ [7.6\%] BEL to BEL pts); $1(0.8 \%)$ pt died in the PBO to BEL group. Proportions of PERR and CRR responders increased from OL baseline to OL Week 28 (Table 1. below)

Proportions of pts who attained SLEDAl scores $<4$ increased from OL baseline to OL Week 28 in the BEL to BEL group and decreased in the PBO to BEL group. Among pts receiving average daily prednisone-equivalent doses of $\leq 5 \mathrm{mg}$ or $\leq 7.5 \mathrm{mg}$ dose was maintained from OL baseline to OL Week 28 (Table 1. below) In pts with autoantibodies at OL baseline, anti-dsDNA and anti-C1q levels decreased from OL baseline to OL Week 28 in both groups. Among pts with low $\mathrm{C} 3 / \mathrm{C} 4$ levels at $\mathrm{OL}$ baseline, $\mathrm{C} 3 / \mathrm{C} 4$ levels increased from $\mathrm{OL}$ baseline to $\mathrm{OL}$ Week 28 in both groups (Table 1. below)

Conclusion: In this OL phase of BLISS-LN, proportions of PERR and CRR responders increased in both the BEL-naïve and BEL-experienced groups; and no new safety signals were observed. Improvements in biomarker levels were observed, especially in pts who switched from PBO to BEL.

Table 1. Responses at OL baseline and OL Week 28 (mITT population, $\mathrm{N}=254$ )

\begin{tabular}{|c|c|c|c|c|}
\hline & \multicolumn{2}{|l|}{ OL baseline* } & \multicolumn{2}{|l|}{ OL Week 28} \\
\hline & $\begin{array}{l}\text { PBO to BEL } \\
(n=122)\end{array}$ & $\begin{array}{l}\text { BEL to BEL } \\
(n=132)\end{array}$ & $\begin{array}{l}\text { PBO to BEL } \\
(n=122)\end{array}$ & $\begin{array}{l}\text { BEL to BEL } \\
(n=132)\end{array}$ \\
\hline $\mathbf{n}$ & 122 & 132 & 118 & 122 \\
\hline PERR, n (\%) & $73(59.8)^{\dagger}$ & $93(70.5)^{\dagger}$ & $79(66.9)$ & $91(74.6)$ \\
\hline CRR, n (\%) & $44(36.1)^{\dagger}$ & $63(47.7)^{\dagger}$ & $57(48.3)$ & 76 (62.3) \\
\hline \multicolumn{5}{|l|}{ SLEDAI score $<4$} \\
\hline $\mathbf{n}$ & 122 & 132 & 120 & 122 \\
\hline Responders, n (\%) & $44(36.1)^{\dagger}$ & $64(48.5)^{\dagger}$ & 40 (33.3) & $64(52.5)$ \\
\hline \multicolumn{5}{|l|}{ Prednisone-equivalent dose } \\
\hline $\mathbf{n}$ & 122 & 132 & 121 & 128 \\
\hline$\leq 5 \mathrm{mg}, \mathrm{n}(\%)$ & $59(48.4)$ & $78(59.1)$ & $60(49.6)$ & 75 (58.6) \\
\hline$\leq 7.5 \mathrm{mg}, \mathrm{n}(\%)$ & $62(50.8)$ & $85(64.4)$ & $66(54.5)$ & $83(64.8)$ \\
\hline \multicolumn{5}{|l|}{ Anti-dsDNA $(\mathrm{IU} / \mathrm{ml})^{\ddagger}$} \\
\hline $\mathbf{n}$ & 85 & 64 & 81 & 61 \\
\hline Median (IQR) levels & $107.0(49.0,212.0)$ & $65.5(42.5,126.5)$ & - & - \\
\hline Median (IQR) \% change from baseline & - & - & $-30.2(-46.3,-6.8)$ & $-10.7(-27.2,9.1)$ \\
\hline \multicolumn{5}{|l|}{ Anti-C1q $(\mathrm{U} / \mathrm{ml})^{\S}$} \\
\hline n & 64 & 60 & 58 & 54 \\
\hline Median (IQR) levels & $71.7(36.6,167.5)$ & $47.1(33.0,75.7)$ & - & - \\
\hline Median (IQR) \% change from baseline & - & - & $-23.0(-41.5,0.5)$ & $-16.5(-33.0,6.1)$ \\
\hline \multicolumn{5}{|l|}{ C3 (mg/dl)" } \\
\hline $\mathbf{n}$ & 45 & 37 & 44 & 35 \\
\hline Median (IQR) levels & $78.0(72.0,83.0)$ & $80.0(71.0,84.0)$ & - & - \\
\hline $\begin{array}{l}\text { Median (IQR) \% change from baseline } \\
\text { C4 (mg/dl)" }\end{array}$ & \multicolumn{3}{|c|}{ C4 (mg/dl) } & $4.7(-4.8,16.9)$ \\
\hline n & 18 & 12 & 18 & 11 \\
\hline Median (IQR) levels & $7.5(6.0,8.0)$ & $7.0(7.0,8.5)$ & - & - \\
\hline Median (IQR) \% change from baseline & - & - & $23.6(11.1,37.5)$ & $11.1(0.0,57.1)$ \\
\hline
\end{tabular}

*DB Week 104 visit and the OL baseline visit were the same visit; ${ }^{\dagger}$ Post hoc analyses; ${ }^{\ddagger}$ Among anti-dsDNA positive pts at OL baseline ( $\left.\geq 30 \mathrm{IU} / \mathrm{ml}\right) ;{ }^{\S} \mathrm{Among}$ anti-C $1 \mathrm{q}$ positive pts at $\mathrm{OL}$ baseline $(\geq 22.2 \mathrm{U} / \mathrm{ml})$; 'Among pts with low C3 $(<90 \mathrm{mg} / \mathrm{dl}) / \mathrm{C} 4(<10 \mathrm{mg} / \mathrm{dl})$ levels at OL baseline. 